\title{
Introducción a los Invariantes de Nudos
}

Pablo F. Ardila ${ }^{1}$

\section{Resumen}

Desde sus orígenes, debidos a los trabajos de Johann Carl Friedrich Gauss y Lord Kelvin, la teoría de nudos ha tenido un problema principal, que consiste en determinar cuándo dados dos diagramas de nudos, estos corresponden al mismo nudo, Peter Tait trató de resolver dicha situación, creando la primera tabla de nudos, sin embargo, no tuvo las herramientas matemáticas para completarla, así hubo la necesidad de establecer mecanismos más precisos para continuar la clasificación de los nudos, esta herramienta se denomina genéricamente invariante. Los invariantes de nudos no son perfectos y sólo sirven cuando queremos ver si dos objetos no son equivalentes, lo cual sucede cuando el mismo invariante es diferente aplicado a dos diagramas, si es igual no se puede concluir nada. Discutiremos sobre los movimientos de Reidemeister, el coloreamiento de un nudo, el polinomio de Alexander, número de enlace, grupo fundamental, además de hablar sobre las aplicaciones en general de la teoría de nudos en diversas ramas del conocimiento. Hacemos una síntesis y exponemos la manera de computar dichos invariantes.

\section{Palaloras clave}

Álgebra, grupo fundamental, invariantes de nudos, movimientos de Reidemeister, polinomio.

1 Docente Auxiliar, INSTITUTO TECNOLÓGICO METROPOLITANO, pabloardila@itm.edu.co

Fecha de recepción: 08 de Noviembre de 2009

Fecha de aceptación: 30 de Septiembre de 2010 


\section{Abstract}

Since its inception, due to the work of Johann Carl Friedrich Gauss and Lord Kelvin, knot theory has had a major problem, that is given is to determine when two diagrams of knots, these correspond to the same knot, Peter Tait tried to resolve the situation, creating the first table of knots, however, did not have the mathematical tools to complete, and there was a need to establish mechanisms needed to continue the classification of knots, this tool is called generically invariant, Knot invariants are not perfect and only work when they want to see if two objects are equivalent, which occurs when the same invariant is applied to two different diagrams, if same cannot conclude anything. This paper has discussed about the most important invariants in knot theory, its history, its mode of use applications. We will discuss the Reidemeister moves, the color of a knot, Alexander polynomial, linking number, fundamental group, in addition to talking about general applications of knot theory in various branches of knowledge. We make a summary and discuss how to compute these invariants.

\section{Keywords}

Algebra, fundamental group, knot invariant, Reidemeister moves, polynomial. 


\section{INTRODUCCIÓN}

Los orígenes de la teoría de nudos se remontan a Carl Friedrich Gauss, quien define inicialmente un nudo como una curva cerrada con varias intersecciones con sí misma. Posteriormente, Lord Kelvin, presenta un modelo atómico, mejorando las ideas de Leucipo y Demócrito, influenciado por los trabajos de Hermann Ludwig Ferdinand von Helmholtz sobre Vórtices y por una publicación de Georg Friedrich Bernhard Riemann (Livingston, 1993), respecto a funciones abelianas, piensa en los átomos como vértices de anillos, y de esta manera las propiedades químicas son relaciones de anudamiento entre átomos, su teoría lo lleva a pensar que lograr clasificar todos los nudos, implicaría conocer toda la estructura atómica, más aún, se podrían clasificar todos los elementos conocidos.

Peter Guthrie Tait, en 1877 publica un primer tratado sobre nudos (Crowell \& Fox, 1963), con la enumeración de los nudos, más tarde, en 1900 conjuntamente con Carl Little, clasifica los nudos hasta 10 cruces, lamentablemente su trabajo tenía un grave problema, el cual era no poder argumentar matemáticamente, el por qué dos nudos eran diferentes (Crowell \& Fox, 1963). Tait usaba como hipótesis de trabajo los siguientes hechos: Un diagrama alternado reducido (sin cruces superfluos) tiene la menor cantidad de cruces para el nudo que representa y dos diagramas alternados reducidos de un nudo dado tienen el mismo índice de nudo.

Hoy en día se conocen como Conjeturas de Tait, y sólo hasta 1980 pudieron ser demostradas usando el Polinomio de Jones. Recientemente, Galatolo y Mieczyslaw, han planteado y resuelto problemas abiertos de la teoría de nudos, tales como el Problema de Montesinos o hallar una función que envíe un nudo no trivial en uno trivial, en la solución de tales problemas se han usado los movimientos de Reidemeister, poderoso invariante de nudos.

La afinidad de la teoría de nudos con otras ramas de las matemáticas, tales como la teoría de integrales matriciales, anillos, espacios vectoriales, es tratada profusamente por Ranicki, Bar-Natan, Abrabsky y Zinn, ellos acoplan las técnicas propias del 
álgebra con miras a definir nuevos invariantes que permitan trabajar de forma más eficaz con nudos.

De creciente desarrollo la topología y el álgebra se constituyen como punto de apoyo, para fundamentar matemáticamente la teoría de nudos, estas dos áreas conjuntas permiten dar una definición rigurosa y precisa de un nudo (Bar-Natan, 2007), pero también sirven para obtener métodos alternativos que ayudan a la solución de muchos problemas que se creían insolubles. En el 2006 Abrabsky, usando las álgebras de temperley-lieb, amplia el marco de acción de la teoría de nudos hacia la física y relaciona esta estructura matemática con un poderoso invariante de nudos conocido como Polinomio de Jones, este descubierto en 1988 sirvió para que su creador V. F. R. Jones, obtuviera la medalla Fields en dicho año (Jones, 2005).

Shaji, Hernerich, Milleet, analizan todo lo relacionado entre la química y la teoría de nudos, haciendo un tratamiento especial a la teoría del DNA y las enzimas, que demuestran tener un comportamiento anudado y por tanto son fácilmente modelables vía teoría de nudos en especial, con la ayuda de invariantes. Kauffman en 1999 crea una interesante vertiente de la teoría de nudos como son los nudos virtuales, y se desata una nueva corriente de preguntas acerca de la importancia de los invariantes, y de la mejor forma de definirlos o adaptarlos.

\section{CONCEPTOS BÁSICOS}

Un nudo es una curva cerrada continua en el espacio Euclideo 3 dimensional $R^{3}$. O de manera análoga usando un poco de geometría, si $p, q$ son dos puntos del espacio, denotemos con $[p, q]$ el segmento de recta que los une. Para un conjunto ordenado de puntos $\left(p_{1}, p_{2}, \ldots, p_{n}\right)$, la unión de los segmentos $\left[p_{1}, p_{2}\right],\left[p_{2}, p_{3}\right] \ldots,\left[p_{n}, p_{1}\right]$ es llamada curva cerrada poligonal. $\mathrm{Si}$ adicionalmente cada segmento se intercepta exactamente en dos puntos cortándose en el punto final, se dice que la curva es simple.

Así que un nudo se puede definir alternativamente como una curva poligonal simple y cerrada en $R^{3}$. El problema de esta definición es que no es aplicable a los llamados nudos salvajes, que 
poseen infinitos cruces. Dado un nudo en el espacio 3 dimensional, su proyección en el plano se conoce como diagrama de un nudo, lo cual facilita su manipulación, si tiene orientación se conoce como diagrama orientado del nudo (ver Fig. 1).

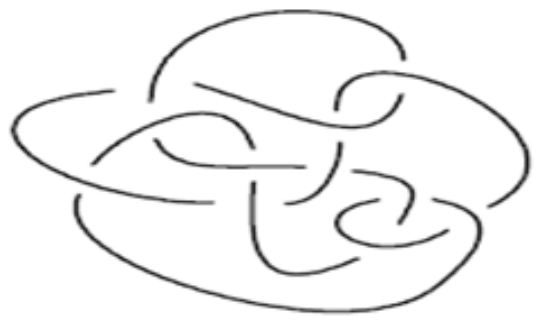

Fig. 1. Diagrama de un nudo de 10 cruces

Surge la pregunta, de cuándo dados dos diagramas de nudos corresponden al mismo, para tal efecto diremos que, dos nudos se dirán equivalentes o del mismo tipo si el uno se puede deformar en el otro, sin efectuar ningún corte. En 1917 el topólogo Max Dehn empleando un método desarrollado por él y conocido como cirugías de Dehn prueba que el trébol derecho e izquierdo son nudos diferentes. Se puede mostrar que si dos nudos tienen la misma proyección en el plano ellos son equivalentes en $R^{3}$.

\section{INVARIANTES DE NUDOS}

Un invariante $\tau$ es un objeto algebraico asociado a diagramas de nudos $D_{1} y D_{2}$. Si dos diagramas de nudos son equivalentes entonces al aplicarle el invariante se tendrá $\tau D_{1}=\tau D_{2}$. Equivalentemente, si $\tau D_{1} \neq \tau D_{2}$ entonces los diagramas de $D_{1}$ y $D_{2}$ no son equivalentes. Para el estudio de los nudos tenemos una serie de métodos combinatorios, los cuales se fundamentan en el estudio de sus diagramas (Birman, 1975), los más importantes son: 


\subsection{Movimientos de Reidemeister}

Creados en 1932 por Kurt Werner Friedrich Reidemeister, y que se resumen en el siguiente teorema: $\mathrm{Si}$ dos nudos son equivalentes, entonces sus diagramas están relacionados por una serie de movimientos de Reidemeister.

Teóricamente, esta herramienta ayuda a distinguir un par de distintos nudos, sin embargo para nudos con diagramas complicados no es tan fácil de aplicar (ver Fig. 2).

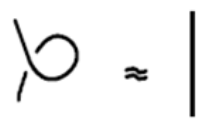

Tipo I.

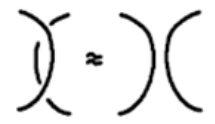

Tipo II.

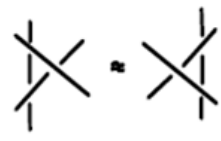

Tipo III.

Fig. 2. Movimientos de Reidemeister de Tipo I, II y III

\subsection{Coloreamiento de un Nudo}

Se emplea para diferenciar nudos usando su Coloreamiento, este concepto fue creado por Ralph Fox. Un nudo es coloreable si cada arco puede pintarse usando uno de tres colores tales que: $\mathrm{Al}$ menos son usados dos colores, y en cada cruce para el cual aparecen dos colores, entonces el tercero aparece.

Se tiene por tanto el siguiente teorema: Si un diagrama de un nudo es coloreable entonces cada diagrama es coloreable. Como ejemplo en la Fig. 3. tenemos el nudo trébol, el cual es coloreable.

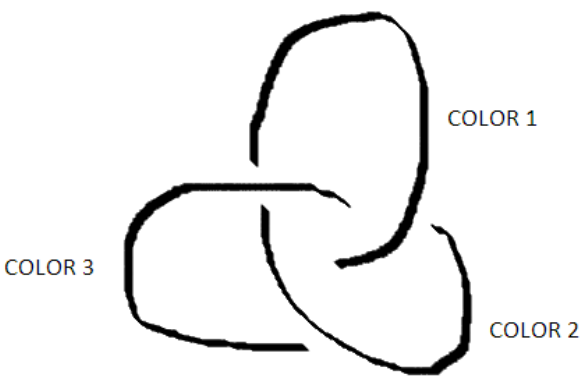

Fig. 3. Nudo 3-coloreable 


\title{
3.3 Polinomio de Alexander
}

En 1938 James Alexander descubre un método por medio del cual le asocia a cada nudo un polinomio, tal que si el cambia el nudo por medio de deformaciones, el polinomio no cambia. Una cantidad que no cambie bajo ciertas transformaciones se denomina invariante. Para el cálculo del Polinomio de Alexander, se siguen los siguientes pasos: Elaborar un diagrama del nudo, Enumerar los arcos del diagrama y Separadamente, enumerar los cruces.

Tome el cruce numerado $L$, si el arco $i$ pasa sobre el arco $j$ y $k$ en el sentido de la mano derecha, entre (1-t) en el puesto $(L, i)$, en la posición $(L, j)$ se coloca $\mathbf{t}$ y en la posición $(L, k)$ poner $\mathbf{- 1}$. Cuando el cruce está orientado en el sentido de la mano izquierda, se pone (1-t) en la posición $(L, i)$, se coloca $\mathbf{t}$ en el puesto $(L, j), \mathrm{y}-\mathbf{1}$ en $(L$, $k$ ), en las demás posiciones de la fila $L$ se entra 0 . Remueva la última fila y columna de la matriz $\mathrm{NxN}$ así obtenida. El determinante de la matriz $(\mathrm{N}-1) \times(\mathrm{N}-1)$ es el Polinomio de Alexander.

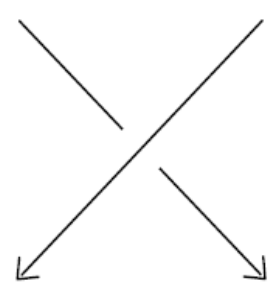

Fig. 4. Cruce derecho e izquierdo

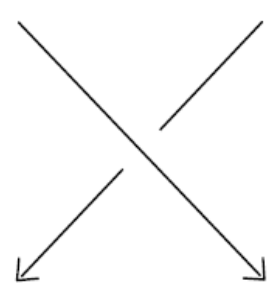

\begin{abstract}
o
\end{abstract}
Aplicando este procedimiento al nudo trébol se obtiene la siguiente matriz

$\left[\begin{array}{ccc}1-t & t & -1 \\ -1 & 1-t & t \\ -1 & t & 1-t\end{array}\right]$

Cuyo determinante luego de eliminar una fila y una columna es: $A(t)=t^{2}-t+1$. 


\subsection{El Grupo Fundamental}

Para un nudo se define como el grupo fundamental de su complemento, es decir es el grupo generado por todas las posibles clases de caminos de homotopía originados en un punto base, se denota por $\pi_{1}\left(R^{3}-T\right)$, donde T es el nudo en cuestión. Calculemos el grupo fundamental de nudo trébol. Comenzamos asignando la relación presentada en la Fig. 5. de acuerdo a como se obtienen los cruces.

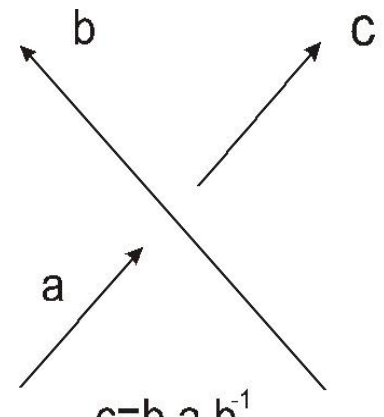

$\mathrm{c}=\mathrm{b}$ a $\mathrm{b}^{-1}$

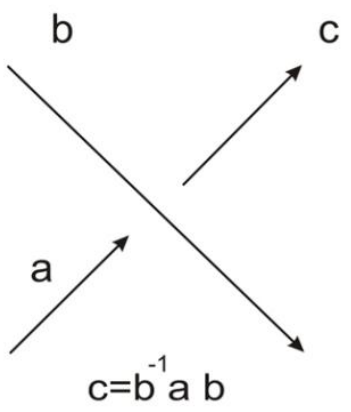

$c=b^{-1} a b$

Fig. 5. Generadores del grupo fundamental

Para continuar tomamos el nudo trébol y le damos una orientación (ver Fig. 6).

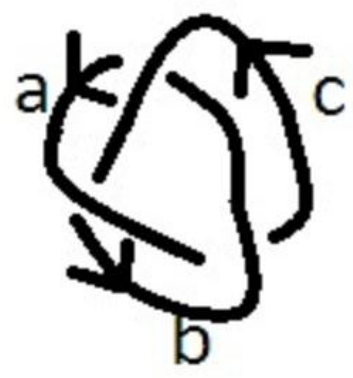

Fig. 6. Nudo trébol con orientación 
En cada cruce se hallan los generadores dados en la Fig. 5, obteniendo las relaciones $a=c b c^{-1}, c=b a b^{-1}, b=a c a^{-1}$.

$\mathrm{Al}$ reemplazar la segunda en la primera se tiene $a b a=b a b$, por tanto el grupo fundamental del nudo trébol es $\pi_{1}\left(R^{3}-T\right)=$ $\{a, b \mid a b a=b a b\}$

\subsection{Mínimo Número de Cruces}

Es la menor cantidad de cruces que se presentan en una proyección de un nudo. El número mínimo número de cruces del nudo de la Fig. 7 es 5.

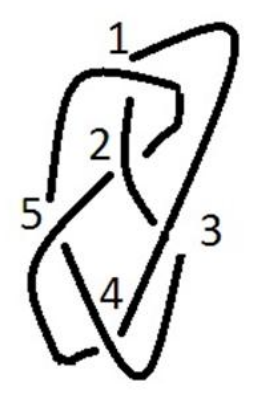

Fig.7. Nudo de 5 cruces

\subsection{El Número de Enlace}

Se usa para enlaces en los que hay más de dos nudos. Se define por (2)

$l(\alpha, \beta)=\frac{1}{2} \sum_{x} \epsilon(x)$

Donde, $\alpha, \beta$ son dos nudos, y $\epsilon(x)$ es +1 o -1 , dependiendo si el cruce es positivo o negativo (o cruce derecho y cruce izquierdo). En el enlace de la Fig. 8. se tiene por (2) que $l(\alpha, \beta)=\frac{1}{2}(-1-1+1+$ $1+1)=\frac{1}{2}$ 


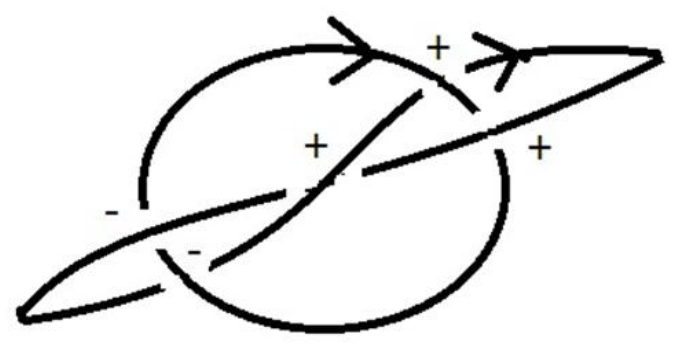

Fig.8. Enlace orientado con dos componentes

\subsection{Desarrollos Finales}

En el año de 1930 Herbert Karl Johannes Seifert, demostró que un nudo es la frontera de una superficie en el 3 espacio dimensional, por tanto las superficies pueden ser usadas para estudiar los nudos, con este descubrimiento queda abierta la puerta para el uso de métodos geométricos en la teoría de nudos.

Empleando métodos geométricos Hermann Schubert's, demostró que existen nudos primos. Christos Papakyriakopoulos en 1957 demuestra el lema de Dehn, que dice que usando métodos geométricos (Dabkowski \& Przytycki, 2004), un nudo no se puede distinguir del nudo trivial, entonces es el nudo trivial.

En 1968 Friedrich Waldhausen probó que dos nudos son equivalentes si y sólo si ciertas propiedades algebraicas son las mismas. Las conjeturas de Tait, se prueban en la década de los 80', empleando para ello un nuevo invariante polinomial llamado el Polinomio de Jones, que usa propiedades de las álgebras de Von Newman.

\section{APLICACIONES}

En 1953 James Dewey Watson y Francis Harry Compton Crick, descubren el ADN, y rápidamente constatan que esta estructura se anuda, por tal motivo se puede modelar empleando la teoría de nudos, así por ejemplo tenemos en la Fig. 9 la imagen del ADN modelada usando un nudo de 6 cruces (Boege \& Hinojosa, 
2006). En los últimos años se ha hecho una clasificación y un trabajo mancomunado entre genetistas y matemáticos para el desarrollo de nuevas tecnologías en el campo de la genética.

De igual forma la química usa los conceptos de teoría de nudos para el trabajo con polímeros, enzimas (Boege \& Hinojosa, 2006) y toda clase de compuestos orgánicos que involucren o tengan una estructura similar a un nudo (Millett, 2010). Recientemente, la mecánica estadística y la computación cuántica se han sumado a la serie de disciplinas que emplean teoría de nudos (Ellgen, 2010).
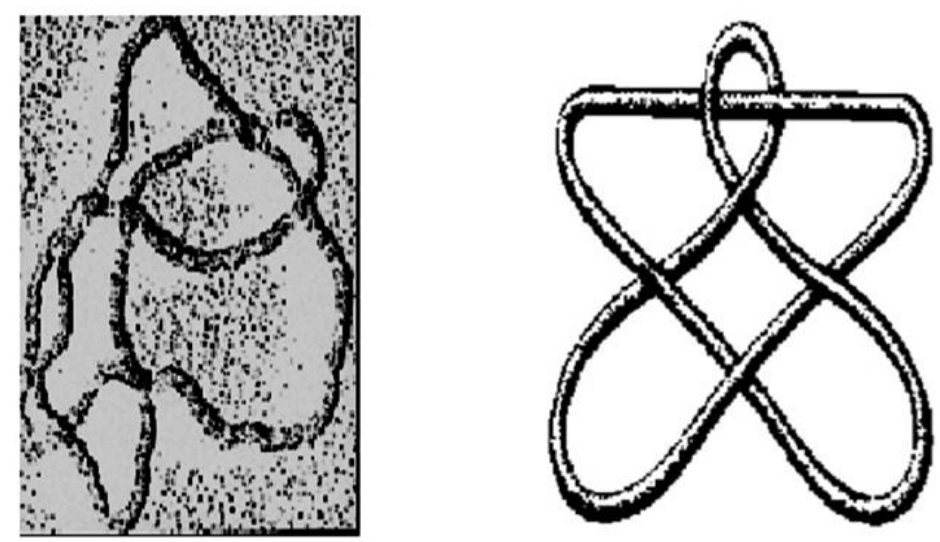

Fig. 9. Imagen del ADN, que corresponde a un nudo de 6 cruces
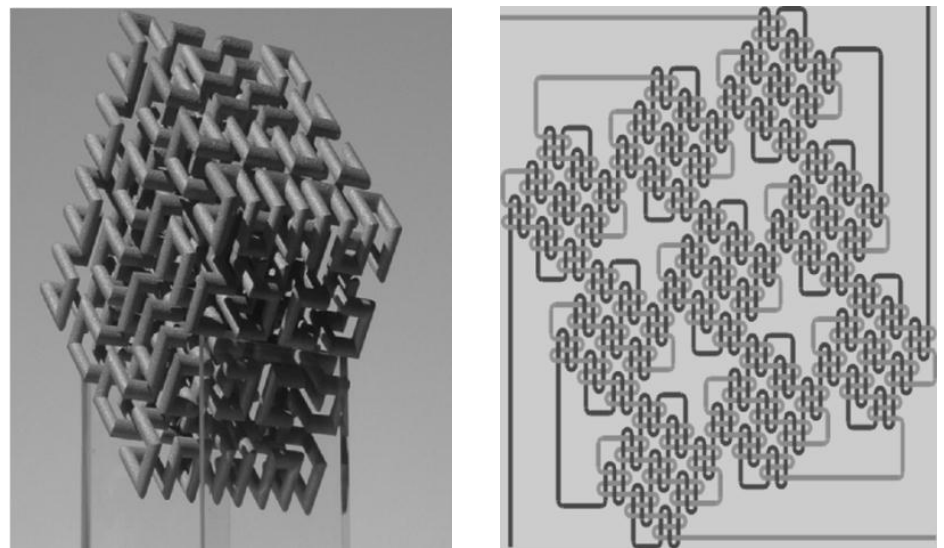

Fig. 10. En la arquitectura y la pintura son recurrentes las formas anudadas 


\section{CONCLUSIONES}

A principios del siglo XX los conceptos elementales de la teoría de nudos se presentaron en forma matemática. Los invariantes matemáticos son objetos que sólo sirven cuando al aplicarlo a dos objetos son diferentes, en este caso los objetos son diferentes. Los invariantes polinomiales más importantes son el Polinomio de Alexander y el Polinomio de Jones. Recientemente se han incorporado invariantes que hacen uso de herramientas topológicas, tales como las superficies de Seifert. Los nudos se aplican en muchas áreas como: la química, la biología, la física, la arquitectura entre otras y dan otras herramientas a científicos deseosos de explorar el micromundo de partículas y células.

\section{REFERENCIAS}

Bar-Natan, D. (2007); Knot Theory and Algebra. http://www.math.toronto.edu/ drorbn/Profile/ResearchProposal-07.pdf

Birman, J., (1975); Braids, Links and Mapping Class Groups, Princenton University Press, New Jersey, USA.

Boege, M., Hinojosa, G., (2006); Nudos para explicar enzimas, Inventio, la génesis de la cultura universitaria en Morelos, $\mathrm{N}^{\circ}$. 4, pp 41-48.

Crowell, R.H., Fox, R.H., (1963); Introduction to Knot Theory, Blaisdell Publishing Company, New York, USA.

Dabkowski, M., Przytycki J., (2004); Unexpected connections between Burnside groups and knot theory, vol. 101 no. 50, pp 17357-17360, PNAS, Boston, USA.

Ellgen, C., (2010); A knot theory of physics, space time in co-dimension 2, www.knotphysics.net

Galatolo, S., (1998); On a problem in effective knot theory, Rend. Mat. Acc. Lincei. s. 9, v. 9:299-306.

Hansen, V., (1989); Braids and Covering, Cambridge University Press, New Jersey, USA. 
Henric, A., (1981); Classical and Virtual pseudodiagram theory and new bounds on unknotting numbers and genus http://arxiv.org/abs/0908.1981

Jones, V.L., (2005); The Jones Polynomial, Department of Mathematics, University of California at Berkeley. http://math.berkeley.edu/ vfr/jones.pdf

Kauffman, L.H., (1999); Virtual Knot Theory, Europ. J. Combinatorics 20, 663-691

Knot Theory: An Introduction

http://www.yucc.yorku.ca/ mouse/knots/intro.html\#what

http://library.thinkquest.org/12295/main.html

http://www.freelearning.com/knots

Livingston, C., (1993); Knot theory, Mathematical Association of America, Washington, DC, USA.

Millett, K., (2010); Physical Knot Theory: An Introduction to the Study of the Influence of Knotting on the Spatial Characteristics of Polymers. WSPC-Proceedings pp.10:56.

Mieczyslaw, K., Dabkowski, Jozef H.P., (2004); Unexpected Connections between Burnside groups and knot theory, PNAS.

Molina, L.A., (2002); Introducción a los Invariantes Polinomiales de Nudos, http://www.matematicas.unal.edu.co/ huerfano/nudos.pdf.

Ranicki, A., (2010); High-dimensional knot Theory, Springer-Verlag. New York, USA.

Shaji, A., (2004); A Brief Introduction to Knot Theory, http://www.cse.iitb.ac.in/ appu/work/knot.pdf.

The Knotplot site http://www.pims.math.ca/knotplot

Zinn, P., Bernard, J., (2010); Knot Theory and Matrix Integrals, http://www.math.buffalo.edu/ menasco/Knottheory.html 